\title{
Fluid Adsorption near an Apex: Covariance between Complete and Critical Wetting
}

\author{
A. O. Parry, M. J. Greenall, and J. M. Romero-Enrique* \\ Department of Mathematics, Imperial College, 180 Queen's Gate, London SW7 2BZ, United Kingdom
}

(Received 4 October 2002; published 29 January 2003)

\begin{abstract}
Critical wetting is an elusive phenomenon for solid-fluid interfaces. Using interfacial models we show that the diverging length scales, which characterize complete wetting at an apex, precisely mimic critical wetting with the apex angle behaving as the contact angle. Transfer matrix, renormalization group, and mean-field analysis show that this covariance is obeyed in 2D and 3D and for long- and short-ranged forces. This connection should be experimentally accessible and provides a means of checking theoretical predictions for critical wetting.
\end{abstract}

DOI: 10.1103/PhysRevLett.90.046101

Advances in the controlled fabrication of micropatterned substrates have stimulated the experimental and theoretical study of fluid adsorption at tailored surfaces [1-5]. For example, Mistura and co-workers [5] have recently investigated complete wetting of Ar on several parallel arrays of wedges and apexes. They show convincingly that the adsorption within the (independent) wedge regions is geometry dominated and distinct from the planar complete wetting case. As well as having implications for microfluidics such studies have also revealed a number of unexpected results relating interfacial fluctuation effects and substrate geometry which have wide application to other phase transitions (see later). In this Letter, we use effective Hamiltonian theory to show that complete wetting on apex shaped substrates reveals a hidden connection (covariance) with critical (continuous) wetting transition occurring on planar surfaces $[6,7]$. The covariance emerges when one considers how, at bulk coexistence, the mean height $l_{A}(\alpha)$ of the unbinding interface above the apex tip depends on the apex angle $\alpha$. For shallow apexes we show that $l_{A}(\alpha)$ is identical to the mean-interfacial thickness occurring at a particular class of critical wetting transition with the apex angle playing the role of an effective contact angle. The covariance is valid for 2D and 3D apexes, for arbitrary intermolecular forces, and is, we believe, a general feature which should also be present in more microscopic models.

The central result of our Letter is the following covariance relation for the interfacial height probability distribution function (PDF) which precisely quantifies the influence of the apex geometry on the complete wetting film. We emphasize that the PDF contains a great deal of information determining the (local) interfacial height, the roughness, and the scaling properties of the density profile. Let $P_{A}^{U}(l, \alpha)$ denote the PDF for the interface height above the apex where the superscript refers to the repulsive binding potential $U(l) \sim B l^{-p}$ for a complete wetting film (see below). Let $P_{\pi}^{V}(l ; \theta)$ denote the PDF for a planar critical wetting transition written in terms of the contact angle $\theta$. Here $V(l)$ is the binding potential for a critical wetting transition which, of necessity, contains
PACS numbers: 68.08.Bc, 05.70.Np, 47.20.-k, 68.35.Md

attractive and repulsive terms. The covariance relation, valid for small $\alpha$ and at bulk coexistence, reads

$$
P_{A}^{U}(l, \alpha)=P_{\pi}^{V}(l ; \alpha),
$$

where the covariant binding potential

$$
V(l)=-A l^{-\chi / 2}+U(l),
$$

with $\chi=\min (p, \tau)$ and $A$ determining the effective contact angle (related to $\alpha$ ). Here $\tau=2(1-\zeta) / \zeta$ and $\zeta$ are the entropic repulsion and interfacial wandering exponents, respectively [8]. Thus the apex locally binds the complete wetting film and induces an effective attractive term in the binding potential, twice the range of the dominant intermolecular or entropic repulsive term. A similar rule applies in 3D for exponentially decaying potentials. Recall that in contrast to abundant experimental studies of complete wetting (including recent work on systems with short-ranged forces [9]) critical wetting transitions are rather rare [6,7] for which no examples are known currently for solid-fluid interfaces. The covariance discussed here provides a means of effectively inducing critical wetting behavior using complete wetting films.

Consider the interface between an infinite apex and a bulk vapor at temperature $T$ and chemical potential $\mu \leq$ $\mu_{\text {sat }}(T)$ (see Fig. 1). We suppose that the flat wall $(\alpha=0)$ is completely wet by the liquid phase at coexistence $\delta \mu \equiv$ $\mu_{\text {sat }}-\mu(T)=0^{+}$corresponding to zero contact angle. The wall shape is described by a height function $z_{A}=$ $-\tan \alpha|x|$ in the $(x, z)$ plane although we shall be interested only in the case of shallow apexes for which we may approximate $\tan \alpha \approx \alpha$. Macroscopically far from the apex tip the height of the interface above the wall is the same as that occurring for a flat wall. Since the liquidvapor interface is required to round the apex, surface tension restrictions imply that the local height $l_{A}$ above the apex tip is smaller and remains finite even in the limit of bulk coexistence. We wish to evaluate the mean-interfacial height $l_{A}(\alpha)$ and interfacial roughness (rms interfacial width) $\xi_{A}(\alpha)$ at bulk coexistence and the 


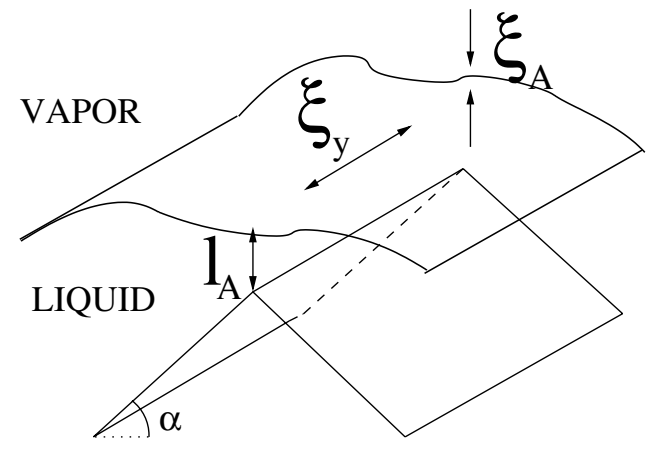

FIG. 1. Schematic illustration of 3D apex complete wetting, showing a section of a typical interfacial configuration above the tip. Diverging length scales are highlighted.

critical exponents

$$
l_{A}(\alpha) \sim \alpha^{-\beta_{A}}, \quad \xi_{A}(\alpha) \sim \alpha^{-\nu_{A}} .
$$

For the 3D apex we also wish to determine the transverse correlation length $\xi_{y}(\alpha) \sim \alpha^{-\nu_{y}}$ pertinent to correlations along the apex. Correlations in the $x$ direction are not described by a finite correlation length and fluctuations are not localized to a region near the apex.

We begin with the 2D apex. The starting point for our calculations is the interfacial Hamiltonian model

$$
\beta H_{A}[l]=\int d x\left\{\frac{\Sigma}{2}\left(\frac{d l}{d x}\right)^{2}+U(l+\alpha|x|)\right\},
$$

where $l(x)$ is the local height of the interface above the $z=0$ reference line, $\Sigma$ is the (reduced) stiffness coefficient (surface tension) of the liquid-vapor interface, and $U(l)$ denotes the binding potential modeling the complete wetting behavior pertinent to the planar system $\alpha=0$. For shallow apexes, it is permissible to assume that the interface interaction with the wall occurs via the relative vertical height $\tilde{l} \equiv l+\alpha|x|$. The binding potential $U(l)$ has an infinite hard wall repulsion and decays as

$$
U(l)=\left(\rho_{l}-\rho_{g}\right) \beta \delta \mu l+B l^{-p},
$$

where, for the moment we have allowed for a finite bulkorder field $\delta \mu>0$. Here $B$ is a positive Hamaker constant while $p$ accounts for the range of the intermolecular forces. Exponentially decaying binding potentials will also be considered in our discussion of the 3D apex. For dimensions in which the free liquid-vapor interface is rough, critical effects at planar complete wetting transitions fall into two classes $[8,10]$ : a mean-field (MF) regime for $p<\tau$ and a fluctuation-dominated regime for $p>\tau$. Heuristically this arises from the interplay between the direct intemolecular repulsion $\sim l^{-p}$ and the effective entropic replusion $U_{\mathrm{fl}}(l) \sim l^{-\tau}$. In the present paper we restrict our attention to pure systems for which the interface is rough for $d \leq 3$ and $\zeta=(3-d) / 2$. For fixed $p$ the upper critical dimension for complete wetting is $d_{\text {co }}=3-4 /(p+2)$ [10].

The model can be studied using transfer matrix methods previously developed for the wedge geometry [11]. Care must be taken in defining an infinite apex geometry and it is convenient to first consider a finite apex extending over the range $[-L / 2, L / 2]$ and impose periodic boundary conditions at the edges. After taking the thermodynamic limit $L \rightarrow \infty$ at finite $\delta \mu>0$ it is straightforward to derive an expression for the interfacial height probability distribution function at arbitrary position $x$ along the wall. At the apex midpoint symmetry considerations simplify this expression considerably

$$
P_{A}^{U}(l ; \alpha) \propto\left|\psi_{0}(l)\right|^{2} e^{-2 \Sigma \alpha l},
$$

where $\psi_{0}(l)$ denotes the ground-state wave function solving the Schrödinger equation

$$
-\frac{1}{2 \Sigma} \psi_{0}(l)^{\prime \prime}+U(l) \psi_{0}(l)=E_{0} \psi_{0}(l)
$$

with boundary conditions $\psi(0)=\psi(\infty)=0$. We now focus on the complete wetting limit $\delta \mu \rightarrow 0$. Macroscopically far from the apex the interface unbinds from the wall. Close to the apex, however, the interface remains bound due to the pinning exponential term in (6). As $\alpha \rightarrow 0$, three different critical behaviors are found: (i) A MF regime for $p<2$, characterized by Gaussian fluctuations with $l_{A}(\alpha) \gg \xi_{A}(\alpha)$; (ii) a marginal case for $p=2$; and (iii) a fluctuation-dominated regime for $p>2$ with universal critical behavior and large scale fluctuations $l_{A}(\alpha) \sim \xi_{A}(\alpha) \sim \alpha^{-1}$. The explicit expressions for the large distance/scaling behavior of $P_{A}^{U}(l ; \alpha)$, determining the critical singularities, are given by

$$
P_{A}^{U}(l ; \alpha) \sim \begin{cases}l^{p / 2} \exp \left(-2 \Sigma \alpha l+\frac{4 \sqrt{2 \Sigma B}}{2-p} l^{1-p / 2}\right), & p<2, \\ l^{1+\sqrt{1+8 \Sigma B}} \exp (-2 \Sigma \alpha l), & p=2, \\ l^{2} \exp (-2 \Sigma \alpha l), & p>2 .\end{cases}
$$

In the MF regime, a saddle point evaluation reveals that

$$
l_{A}(\alpha) \sim\left(\frac{2 B}{\Sigma \alpha^{2}}\right)^{1 / p}
$$

$\nu_{A}=1 / p+1 / 2$. The critical exponents are continuous at $p=2$. These results completely classify the asymptotic critical behavior for complete wetting at a $2 \mathrm{D}$ apex in pure systems. At this point we make two remarks:

(A) The values of the critical exponents follow from a simple mean-field/entropic repulsion argument. Ignoring fluctuations the equilibrium interfacial profile is obtained from minimization of the effective Hamiltonian. A first integral of the Euler-Lagrange equation determines the midpoint height at bulk coexistence according to 


$$
\frac{\Sigma \alpha^{2}}{2}=U\left(l_{A}\right)
$$

and leads directly to the result (9) valid in the MF regime $(p<\tau)$. For $p>\tau$ interfacial wandering leads to an entropic repulsion $U_{\mathrm{fl}} \sim l^{-\tau}$. Thus we should expect two regimes with $\beta_{A}=\max [2 / p, \zeta /(1-\zeta)]$ in agreement with the explicit calculation for $\zeta=1 / 2$. For later purposes observe that the MF Eq. (10) is also appropriate for higher dimensional apexes.

(B) The PDF's and associated critical exponents are identical to those occurring at a certain class of 2D critical wetting transition. At a critical wetting transition the mean height of the interface $l_{\pi}$, roughness $\xi_{\perp}$, and parallel correlation length $\xi_{\|}$for a planar substrate diverge as the temperature (say) is increased towards a wetting temperature $T_{w}\left(\mu=\mu_{\text {sat }}\right)$. This is equivalent to the contact angle $\theta$ of a sessile drop vanishing as $T \rightarrow$ $T_{w}^{-}$. The standard interfacial model for this is

$$
\beta H_{\pi}[l]=\int d x\left\{\frac{\Sigma}{2}\left(\frac{d l}{d x}\right)^{2}+V(l)\right\},
$$

where $V(l)$ denotes an appropriate binding potential. The associated $\operatorname{PDF} P_{\pi}^{V}(l ; \theta)$ can be calculated using standard methods which map the problem onto one dimensional quantum mechanics [10]. In particular, consider 2D critical wetting transitions occurring for the class of potentials (2) with $\chi=\min (p, 2)$. For such potentials calculations show that $A \propto \theta$. A straightforward calculation of the PDF's $P_{\pi}^{V}(l ; \theta)$ for $p<2, p=2$, and $p>2$ yields results that, in the critical limit (small $\theta$ ), are identical to (8) provided we set $\theta=\alpha$ [12]. Apex complete wetting precisely mimics the properties of a critical wetting transition. From the covariance of the PDF's it follows that the mean-interfacial heights satisfy

$$
l_{A}(\alpha)=l_{\pi}^{V}(\alpha),
$$

where the right-hand side is understood to represent the mean height at a planar critical wetting transition with the covariant potential (2). It is notable that apex covariance is obeyed at MF level and beyond, and therefore not necessarily related to fluctuation induced effects such as hyperscaling. In particular, the relation (12) follows directly from comparing the solution of the MF Eq. (10) with the position of the minimum of the critical wetting potential (2) (with $\chi=p$ ). The covariance relations for apex complete wetting are similar, but not identical, to those which exist for 2D wedge filling transitions. For both pure and impure systems 2D filling transitions mimic the properties of planar critical wetting transitions with short-ranged forces in contrast to the present apex problem where the equivalent critical wetting transition has long(er)-ranged forces. Wedge covariance and filling are closely related to the Indekeu-Robledo conjecture for the line tension [13], and the unzipping transition for stranded polymer chains [14]. It is likely that similar connections may also apply for the apex geometry.

Having discussed the 2D apex in detail it is straightforward to generalize the results to $3 \mathrm{D}$ systems based on the interfacial model

$$
\beta H_{A}[l]=\int d \mathbf{x}\left\{\frac{\Sigma}{2}(\nabla l)^{2}+U(l+\alpha|x|)\right\},
$$

where for purposes of generality we have written $\mathbf{x}=$ $\left(x, \mathbf{x}_{\|}\right)$, where $\mathbf{x}_{\|}$denotes the $d-2$ dimensional vector along the apex axis. In $3 \mathrm{D} \mathbf{x}=(x, y)$ but it is instructive to also consider the generalized apex for $2 \leq d<3$ since this gives clear indication that the covariance relations extend to higher dimensions. First rewrite the Hamiltonian in terms of the relative height $\tilde{l}$. The critical behavior follows from elementary renormalization group (RG) considerations. Under rescaling $\mathbf{x} \rightarrow \mathbf{x}^{\prime}=\mathbf{x} / b, l \rightarrow$ $l^{\prime}=l b^{-\zeta}$ the renormalized tilt angle and Hamaker constant are $\alpha^{\prime}=\alpha b^{1-\zeta}$ and $B^{\prime}=B b^{2-\zeta p-2 \zeta}$, respectively. Thus $\alpha$ is always a relevant scaling field while the intermolecular forces are only relevant for $p<\tau$. The criticality falls into two scaling regimes consistent with the explicit 2D results: (i) A MF regime for $p<\tau$ with $\beta_{A}=$ $2 / p, \nu_{y}=2 / p+1$, and $\nu_{A}=\zeta \nu_{y}$ for which (10) is valid. (ii) A fluctuation regime for $p>\tau$ describing the universality class of systems with short-ranged forces with $\beta_{A}=\zeta /(1-\zeta), \nu_{y}=1 /(1-\zeta)$, and $\nu_{A}=\zeta \nu_{y}$.

Note that for fixed $p$ the upper critical dimension $d_{A}=$ $3-4 /(p+2)$ and is unchanged from the planar complete wetting result $d_{c o}$. Remarks (A) and (B) made earlier about the $2 \mathrm{D}$ results also apply in higher dimensions, where the planar covariant effective Hamiltonian is

$$
\beta H_{\pi}[l]=\int d \mathbf{x}\left\{\frac{\Sigma}{2}(\nabla l)^{2}+V(l)\right\} .
$$

For the physically relevant case $d=3$, MF theory is valid for all long-ranged intermolecular forces (finite $p$ ). Thus for nonretarded van der Waals forces we predict $l_{A}(\alpha) \sim \sqrt{2 B / \Sigma \alpha^{2}}$ and observe that this is identical (covariant) with the growth of the interfacial thickness at a critical wetting transition with binding potential $V=$ $-A / l+B / l^{2}$. Similar remarks apply for the interfacial roughnesses at the respective transitions $\left[\xi_{A}(\alpha) \sim \xi_{\perp} \sim\right.$ $\sqrt{-\ln \alpha}$ ]. Our final task is to address the issue of covariance for the marginal case of 3D systems with shortranged forces. For this we use the interfacial model (13) with binding potential $U=B \exp (-\kappa l)$ and $\kappa$ the inverse bulk correlation length. At MF level we find $\kappa l_{A}(\alpha) \sim$ $-2 \ln \alpha$ while solution of the Ornstein-Zernike equation for the height-height correlation function along the apex tip yields $\xi_{y} \sim \alpha^{-1}$ [15]. Thus the MF exponents for short-range forces are $\beta_{A}=0(\ln ), \nu_{y}=1$ and are consistent with the $\zeta \rightarrow 0$ limit of the short-ranged exponents detailed in (ii). Beyond MF we anticipate that $\nu_{y}$ is unchanged but that the logarithmic divergence of $l_{A}$ is 
altered. To study this we employ the same approximate linear functional RG approach used for the 3D planar wetting transition but with an appropriately modified matching condition for the new geometry [16]. Under the rescaling of $l$ and $\mathbf{x}$ described earlier (with $\zeta=0$ ) the binding potential maps as $U(l) \rightarrow R^{(b)}[U]$ which effectively coarse grains the potential over the interfacial roughness. Since the angle and correlation length rescale as $\alpha^{\prime}=b \alpha$ and $\xi_{y}^{\prime}=\xi_{y} / b$ one can curtail the renormalization at $b \sim \alpha^{-1}$ at which scale both the renormalized angle and transverse correlation length are of order unity and fluctuation effects are negligible. Matching with MF theory implies $\Sigma / 2=R^{(1 / \alpha)}\left[U\left(l_{A}\right)\right]$ and a simple calculation yields

$$
\kappa l_{A}(\alpha) \sim-(2+\omega) \ln \alpha,
$$

where $\omega=\kappa^{2} /(4 \pi \Sigma)$ is the usual wetting parameter and we have assumed $\omega<2$ as pertinent to the bulk Ising universality class. This critical behavior is again consistent with covariance as can be seen by comparison with the 3D short-ranged critical wetting transition described by the model (14) with potential

$$
V(l)=-A e^{-(\kappa l / 2)}+B e^{-\kappa l}, \quad l>0,
$$

where, guided by our earlier findings, we have included an attractive term which is twice the range of the direct repulsion. This is the same binding potential appearing in the standard theory of short-ranged critical wetting except for a trivial factor of 2 in the definition of the inverse bulk correlation length. Accordingly, rescaling $\kappa$ and $\omega$ in the known RG results for critical wetting [16]

$$
\kappa l_{\pi}^{V}(\theta) \sim-(2+\omega) \ln \theta,
$$

where the (rescaled) wetting parameter $\omega<2$. Note also that the divergence of $\xi_{y} \sim \alpha^{-1}$ is similar to the behavior of the critical wetting transverse correlation length written in terms of the contact angle $\xi_{\|}(\theta) \sim \theta^{-1}$. Interestingly one still finds the critical behavior (15) if one improves the apex calculation to account for a positiondependent stiffness coefficient [17]. That is, even if planar short-ranged wetting transitions are driven first-order by a stiffness instability mechanism, the apex still mimics the properties of critical wetting.

We finish with comments relevant to experimental studies on periodic systems. Close to bulk coexistence the interfacial height above a single, infinite apex shows scaling behavior $l_{A} \sim \alpha^{-\beta_{A}} W_{(1)}\left(\delta \mu \alpha^{-\Delta_{A}}\right)$ with gap exponent $\Delta_{A}=2+\beta_{A}$. On a periodic array (with wavelength $L$ ) finite size effects modify this to $l_{A} \sim \alpha^{-\beta_{A}} W_{(2)}\left(\delta \mu \alpha^{-\Delta_{A}}, L / \xi_{\|}^{\mathrm{co}}\right)$, where $\xi_{\|}^{\mathrm{co}} \sim \delta \mu^{-\nu_{\|}^{\mathrm{co}}}$ is the transverse correlation length for planar complete wetting. Thus the adsorption above the apex tip only behaves like a single apex for sufficiently large $L \gg$ $\xi_{\|}^{\text {co }}$. This is equivalent to the requirement that the vertical distance between the apex tip and the wedge trough, $\alpha L / 2$, is much larger that the local height of the interface above the wedge bottom $l_{w} \sim \Sigma \alpha^{2} / 2 \beta \delta \mu\left(\rho_{l}-\rho_{g}\right)$.

In this Letter, we have shown that complete wetting at an apex mimics precisely planar critical wetting. Taken together with similar covariance relations for wedge filling, there is clear evidence of a fundamental connection between contact and geometric angles. Further work is required to understand such covariances at a deeper level.

J. M. R. -E. and M. J. G. acknowledge financial support from Secretaría de Estado de Educación y Universidades (Spain), cofinanced by the European Social Fund, and EPSRC (U.K.), respectively.

*On leave from Departamento de Física Atómica, Molecular y Nuclear, Area de Física Teórica, Universidad de Sevilla, Apartado de Correos 1065, 41080 Sevilla, Spain.

[1] S. Dietrich, in New Approaches to Old and New Problems in Liquid-State Theory, edited by C. Caccamo et al., NATO Advanced Study Institutes, Ser. B (Kluwer, Dordrecht, 1998), p. 197.

[2] H. Gau et al., Science 283, 46 (1999).

[3] C. Rascón and A. O. Parry, Nature (London) 407, 986 (2000).

[4] S. Gheorghiu and P. Pfeifer, Phys. Rev. Lett. 85, 3894 (2000).

[5] L. Bruschi, A. Carlin, and G. Mistura, J. Chem. Phys. 115, 6200 (2000); Phys. Rev. Lett. 89, 166101 (2002).

[6] For a general review of wetting, see, for example, S. Dietrich, in Phase Transitions and Critical Phenomena, edited by C. Domb and J. L. Lebowitz (Academic Press, New York, 1988), Vol. 12, p. 1.

[7] For recent experimental reviews, see D. Bonn and D. Ross, Rep. Prog. Phys. 64, 1085 (2001); B. M. Law, Prog. Surf. Sci. 66, 159 (2001).

[8] M. E. Fisher, J. Chem. Soc., Faraday Trans. 2 82, 1569 (1986).

[9] P. Huber et al. Phys. Rev. Lett. 89, 035502 (2002).

[10] R. Lipowsky, Phys. Rev. B 32, 1731 (1985).

[11] A. O. Parry, M. J. Greenall, and A. J. Wood, J. Phys. Condens. Matter 14, 1169 (2002).

[12] The exact covariant potential $V=-\alpha \psi_{0}^{\prime} / \psi_{0}+U$ reduces to Eq. (2) for large $l$.

[13] J. O. Indekeu and A. Robledo, Phys. Rev. E 47, 4607 (1993).

[14] D. K. Lubensky and D. R. Nelson, Phys. Rev. Lett. 85, 1572 (2000).

[15] Further details will be published elsewhere.

[16] D. S. Fisher and D. A. Huse, Phys. Rev. B 32, 247 (1985).

[17] M. E. Fisher and A. J. Jin, Phys. Rev. Lett. 69, 792 (1992). 